Manuscript post-print for self-archiving purposes.

Optics Letters 35, 3348 (2010)

doi: $10.1364 /$ OL.35.003348

\title{
Thermal Emission of Macroporus Silicon Chirped Photonic Crystals
}

\author{
Moisés Garín*1,2,3, Trifon Trifonov ${ }^{3,4}$, David Hernández ${ }^{3}$, Ángel \\ Rodriguez ${ }^{3}$, and Ramón Alcubilla ${ }^{3,4}$ \\ ${ }^{1}$ Centro de Tecnologías Físicas, Unidad asociada ICMM-CSIC/UPV, Universidad \\ Politécnica de Valencia, Av. de los Naranjos s/n., 46022 Valencia, Spain \\ ${ }^{2}$ Instituto de Ciencia de Materiales de Madrid (CSIC), Cantoblanco, 28049 \\ Madrid, Spain \\ ${ }^{3}$ Departament d'Engingeria Electrònica, Universitat Politècnica de Catalunya,, \\ c/Jordi Girona 1-3 Mòdul C4, 08034 Barcelona, Spain \\ ${ }^{4}$ Center for Reasearch in Nanoengineering CRnE (UPC), c/Pascual $i$ Vila 15, \\ 08028 Barcelona, Spain
}

\begin{abstract}
In this article we report on the thermal properties of macroporous silicon photonic crystals with the unit cell gradually varied along the pore axis. We show experimentally that arbitrarily large omnidirectional total reflectance bands can be produced with such structures. We also demonstrate that those bands can be effectively used to reduce thermal radiation in large spectral bands.
\end{abstract}

\section{Introduction}

The capability to engineer materials that concentrate thermal radiation in a desired wavelength region, the so-called selective thermal emitters, is of paramount importance in order to raise efficiency in areas such as lighting [1] and thermophotovoltaic energy conversion [2].

Photonic band gap (PBG) materials [3] promise to be excellent candidates for the development of selective emitters due to their ability to control and confine light. It has been demonstrated that thermal radiation from a PBG structure can be both fully suppressed inside the band gap [4] or enhanced outside it 2] due to the high redistribution of the photonic density of states. Nevertheless, the fabrication of

\footnotetext{
*Corresponding author: moises.garin@upc.edu
} 
large-area high-quality PBG structures able to remain stable at high temperatures still poses a challenge.

A possible choice material for tailoring thermal emission is three-dimensional macroporous silicon (MP-Si). Macroporous Si [5] is a versatile material formed by photo-assisted electrochemical dissolution of silicon in hydrofluoric (HF) acid solution. In those structures, the pore sites are defined through standard lithographic techniques while periodicity in the third dimension is introduced by modulating the photocurrent during the pore growth [6]. In this way, full-wafer-surface 3D structures, with as many periods in the pore axis as allowed by the wafer thickness, can be produced. In addition, since the remaining silicon is crystalline, these structures can withstand temperatures closer to the melting point of silicon without deformation.

Crystalline silicon is an excellent IR emitter when doped or at high temperatures, exhibiting a constant emissivity of around 0.7. For this reason, the capability to suppress thermal emission is essential in order to fabricate efficient selective emitters based on MP-Si photonic crystals. Up to now, it has been reported that thermal emission can be fully suppressed from the surface of 3D MP-Si structures thanks to the PBG effect [7, 8]. Although these structures do not feature a complete 3D PBG but in certain directions, they do build an omnidirectional total reflection band able to supress thermal radiation from the sample's top surface. Unfortunately, this effect is restricted to somewhat narrow frequency ranges, typically up to a $10 \%$ of the midband frequency [8]. Beyond that limitation, the flexibility of macroporous silicon can be exploited in order to design pore morphologies featuring omnidirectional external-reflectivity bands in wider wavelength regions. In particular, Yablonovitch suggested in [9] that arbitrary large omnidirectional external-reflectivity bands can be created through chirped Bragg reflectors, where the layer spacing is increased smoothly along the crystal. Porous materials created by electrochemical techniques are excellent candidates for fabricating chirped structures, since the porosity profile can be almost freely selected. Specifically, chirped bragg mirrors based on microporous silicon have been already produced [10, 11].

In this article we report on the thermal emission characteristics of chirped MP-Si structures in the MID-IR wavelength region $(10-30 \mu \mathrm{m})$. Very large external totalreflectance bands, spanning from $12 \mu \mathrm{m}$ to $27 \mu \mathrm{m}$ is experimentally demonstrated. Experimental thermal emission measurements demonstrates that thermal emission is strongly reduced in that range.

\section{Experimental}

A detailed description of the sample preparation and electrochemical etching can be found elsewhere [12, the following is a brief summary of the process. Base material is $\langle 100\rangle$ oriented, single side polished, $n$-type, crystalline silicon wafers with a resistivity of $1-10 \Omega \mathrm{cm}$. As a transparent ohmic contact, a thin $\mathrm{n}^{+}$layer is implanted on the backside of the wafer. First, the nucleation sites of the pores are defined by patterning a square array of inverted pyramids with a periodicity, $a$, of $4 \mu \mathrm{m}$. Then, the sample is electrochemically etched under backside illumination in aqueous HF solution ( 5 wt \%) with a small amount of ethanol as wetting agent.

During the electrochemical process, the etching current is varied periodically 
following a sawtooth-like profile in order to obtain sinusoidal-like modulated pores [6]. In addition, to form the chirp structure, the pore modulation length, $L_{z}$, is increased period by period following the expression $L_{z}(i)=L_{z 0}+\left(L_{z f}-L_{z 0}\right)(i-1)(N-1)^{-1}$, where $L_{z 0}$ and $L_{z f}$ are the intial (surface) and final modulation length, respectively, $i=1, \ldots, N$ is the period number, and $N$ is the total number of periods, The maximum and minimum pore diameters are constant throughout all sample periods.

After fabrication, samples underwent a pore widening process based on several oxidation/oxide removal steps in order to optimize the pore's structure [13, 8]. Approximately $260 \mathrm{~nm}$ of pore wall was eroded, enough to interconnect the pores laterally, producing a 3D-like structure. As an example, Figure 1 shows a SEM picture of a sample with $L_{z 0}=4 \mu \mathrm{m}, L_{z f}=5 \mu \mathrm{m}$ and $N=35$. The picture has been taken from a bird's eye point of view where the surface and the cleaved side can be seen at the same time. It must be emphasized that, In spite of 3D-like structure, the external total reflectance feature of interest can be understood with a simplified 1D effective model. Under that approximation, the pore widening process enhances the refractive index contrast and, at the same time, shifts the features toward shorter wavelengths due to the lower average refractive index.

In a smooth chirped macroporous structure, i.e. with slight changes in $L_{z}$ between adjacent periods, light of a particular wavelength travels through the structure until it meets a region with $L_{z}$ fulfilling the Bragg condition, where it is reflected. The external reflectance band is, thus, defined by $L_{z 0}$ and $L_{z f}$ and can be extended arbitrarily, provided that loses are negligible. As a proof of concept we have fabricated several samples with different initial and final periodicities (e.g. $3-6 \mu \mathrm{m}$, 4-6 $\mu \mathrm{m}$ and $4-5 \mu \mathrm{m}$ ) and with a total number of periods ranging from 21 to 41 periods, keeping the rest of parameters the same. The total thickness of these structures range between $100 \mu \mathrm{m}$ and $200 \mu \mathrm{m}$, approximately. In fact, the modulation length cannot be much smaller than the in-plane periodicity [14]. Actually, $3 \mu \mathrm{m}$ is already the minimum $L_{z}$ that we can effectively achieve. Notice also that the average porosity is independent of $L_{z}$ and, therefore, it is constant throughout the sample.

The external total reflectance band of the prepared samples has been characterized through angle-resolved reflectance measurements using a Bruker's Vertex 70 Fourier Transform Infrared Spectrometer (FT-IR) equiped with a variable-angle specular-reflection accesory A513/QA. The setup allowed incidence angles from quasi-normal $\left(13^{\circ}\right)$ up to nearly gracing incidence angle $\left(83^{\circ}\right)$, while the opening angle of the incident beam is $\pm 6^{\circ}$, which suffices for the smooth angle dependece of the features of interest. In figure 2 we show the reflectance results for a sample with periodicity chirped from 3 to $6 \mu \mathrm{m}$ and 41 periods. A gold mirror was used as reference. Fig. 2(a) shows the reflectance map while fig. 2(b) is the quasi-normal reflectance spectra. As it can be seen, an extraordinarily wide high reflectance band appears for all incidence angles. An interesting feature seen in the spectrum is a series of low reflectance peaks that diminish the average reflectance of the high reflectance band. These peaks, initially unexpected, originate from Fabry-Perot-like resonant coupling of the incident wave, therefore reducing the reflectance. Increasing the number of periods, i.e. a smoother $L_{z}$ variation, increases the density of resonant peaks but makes them shallower as more periods are helping to reflect 
those wavelengths.

Owing to the Kirchhoff's law, stating that $\epsilon=1-r$, we expect thermal emission to be considerably reduced inside the enlarged total reflectance band exhibited by the chirped structures. To demonstrate it, we have experimentally measured the emissivity of the structures using a Vertex 70 FT-IR spectrometer equipped with the A540 emission adapter. This commercial set-up allows normal thermal emission measurement, with a beam opening angle of $\pm 7.5^{\circ}$, from room-temperature up to $400{ }^{\circ} \mathrm{C}$. In this set-up, the sample is clamped vertically to a metallic surface which is at a constant temperature. After thermalisation the sample is at a constant and homogeneous temperature very close to the heater temperature, since c-Si is an excellent thermal conductor. In spite of that, a small (and unknown) temperature gradient in the pore axis is unavoidable, specially at temperatures above $200{ }^{\circ} \mathrm{C}$, as the sample is heated by the back. Sample's emissivity values are obtained after conducting a standard two-temperature calibration using a piece of polished Si covered with a black organic film $(\epsilon=95 \%)$ as reference.

Figure 3 (a) and (b) shows measured emissivity spectra for two samples with $L_{z}=3-5 \mu \mathrm{m}$ identical except for the number of periods. As expected, the thermal emission is greatly reduced in the high reflectance band, but the $\epsilon$ increases rapidly at longer wavelengths at high temperatures specially for the sample with a longer number of periods. This is, in fact, not surprising as longer wavelengths must travel longer distances before being reflected, whereas shorter wavelengths are reflected near the surface. As a result, the increased loses of silicon at higher temperatures has a stronger effect at longer wavelengths. In general, a long number of periods enhances the reflectance at room-temperature but punishes the emissivity at high temperatures and, therefore, a compromise must be reached.

\section{Conclusion}

In summary, large external-total-reflection bands have been achieved by using macroporous silicon structures with a chirped modulation period. In particular, a reflectance band expanding from wavelengths from 11 up to $25 \mu \mathrm{m}$ has been experimentally demonstrated. It has been also shown that these structures effectively reduce the thermal emission in that band. In conclusion, it has been demonstrated that chirped macroporous silicon structures can block thermal radiation in wider bands than regular structures.

\section{Acknowledgements}

This work has been funded by the Spanish Government through CICYT-TEC200767081 and CICYT-TEC2008-02520.

\section{References}

[1] J. F. Waymouth, U.S. Patent No. 5,079,473 (Jan. 7, 1992). 
[2] S. Y. Lin, J. Moreno, and J. G. Fleming, Appl. Phys. Lett. 83, 380-382 (2003).

[3] E. Yablonovitch, Phys. Rev. Lett. 58, 2059-2062 (1987).

[4] S.-Y. Lin, J. G. Fleming, E. Chow, J. Bur, K. K. Choi, and A. Goldberg, Phys. Rev. B 62, R2243-R2246 (2000).

[5] V. Lehmann, J. Electrochem. Soc. 140, 2836-2843 (1993).

[6] J. Schilling, F. Müller, S. Matthias, R. B. Wehrspohn, U. Gösele, and K. Busch, Appl. Phys. Lett. 78, 1180-1182 (2001).

[7] M. Garín, T. Trifonov, A. Rodríguez, and R. Alcubilla, Appl. Phys. Lett. 91, 181901 (2007).

[8] M. Garín, T. Trifonov, A. Rodríguez, R. Alcubilla, F. Marquier, C. Arnold, and J.-J. Greffet, Appl. Phys. Lett. 93, 081913 (2008).

[9] E. Yablonovitch, Opt. Lett. 23, 1648-1649 (1998).

[10] I. J. Kuzma-Filipek, F. Duerinckx, E. V. Kerschaver, K. V. Nieuwenhuysen, G. Beaucarne, and J. Poortmans, J. Appl. Phys. 104, 073529 (2008).

[11] E. Xifré-Pérez, L. F. Marsal, J. Pallarès, and J. Ferré-Borrull, J. Appl. Phys. 97, 064503 (2005).

[12] T. Trifonov, M. Garín, A. Rodríguez, L. F. Marsal, and R. Alcubilla, Phys. Status Solidi A 204, 3237-3242 (2007).

[13] S. Matthias, F. Müller, C. Jamois, R. B. Wehrspohn, and U. Gösele, Adv. Mater. 16, 2166-2170 (2004).

[14] J. Schilling, F. Müller, R. B. Wehrspohn, U. Gösele, and K. Busch, in "Materials and Devices for Optoelectronics and Microphotonics MRS Proceedings Volume 722," (Materials Research Society, 2002), paper L6.8. 


\section{List of Figures}

$1 \quad$ SEM picture of a macroporous chirped sample with periodicity varied from $4 \mu \mathrm{m}$ to $5 \mu \mathrm{m}$ in 35 periods. . . . . . . . . . . . . . . 7

2 (Color online) (a) Experimental reflectance map of a chirped sample with $L_{z}=3-6 \mu \mathrm{m}$ in 41 periods. The colorbar indicates the reflectance scale, with red and blue being high and low reflectance values, respectively. (b) Reflectance spectrum at quasi-normal incidence. . . . . . . 8

3 Measured normal emissivity spectra of two chirped samples with $L_{z}=3-5 \mu \mathrm{m}$ and (a) 21 periods and (b) 41 periods. Emissivity is measured at temperature steps of $50{ }^{\circ} \mathrm{C}$ in the direction of the arrow. $\quad 9$ 


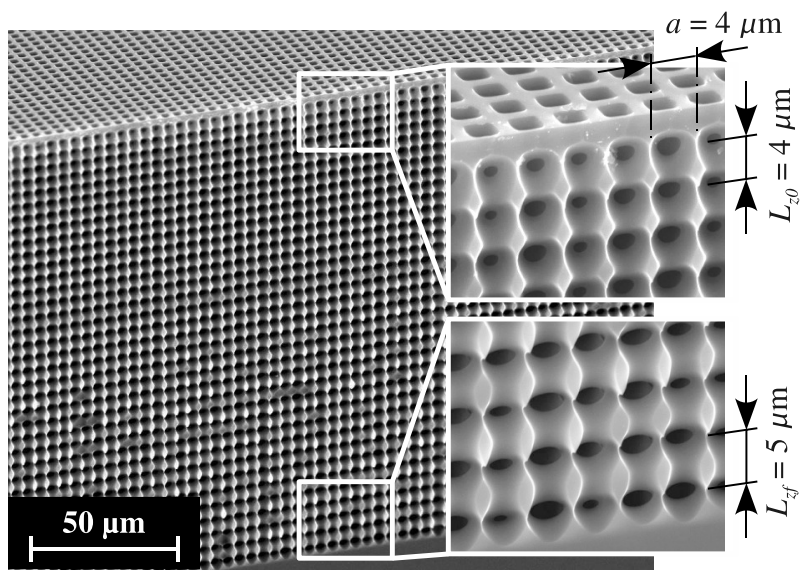

Figure 1: SEM picture of a macroporous chirped sample with periodicity varied from $4 \mu \mathrm{m}$ to $5 \mu \mathrm{m}$ in 35 periods. 


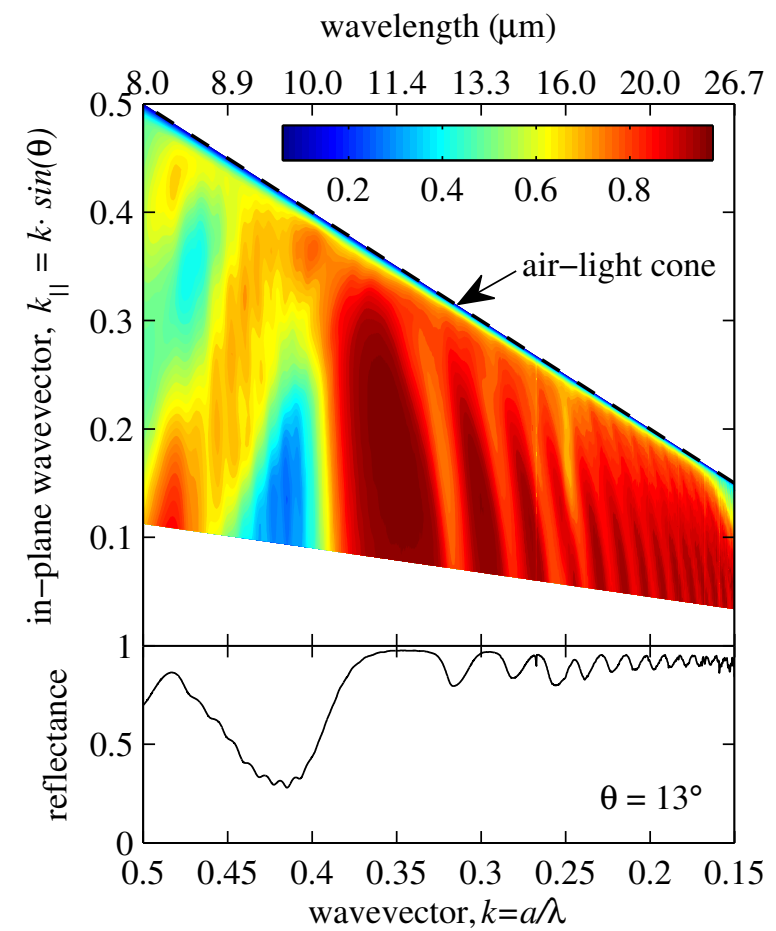

Figure 2: (Color online) (a) Experimental reflectance map of a chirped sample with $L_{z}=3-6 \mu \mathrm{m}$ in 41 periods. The colorbar indicates the reflectance scale, with red and blue being high and low reflectance values, respectively. (b) Reflectance spectrum at quasi-normal incidence. 


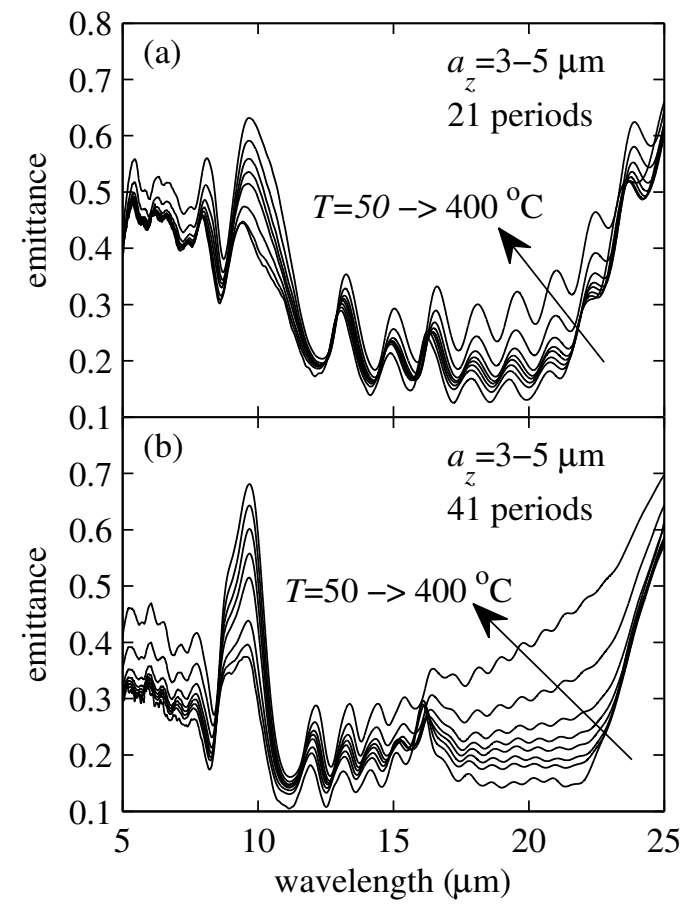

Figure 3: Measured normal emissivity spectra of two chirped samples with $L_{z}=3-$ $5 \mu \mathrm{m}$ and (a) 21 periods and (b) 41 periods. Emissivity is measured at temperature steps of $50{ }^{\circ} \mathrm{C}$ in the direction of the arrow. 